\title{
Extracellular Vesicles in Psychiatry Research in the Context of RDoC Criteria
}

\author{
Can Ilgın $\bowtie$ and Ahmet Topuzoğlu \\ Department of Public Health, Marmara University School of Medicine, Istanbul, Turkey
}

\begin{abstract}
The analysis of extracellular vesicles has been accelerated because of the technological advancements in omics methods in recent decades. Extracellular vesicles provide multifaceted information regarding the functional status of the cells. This information would be critical in case of central nervous system cells, which are confined in a relatively sealed biological compartment. This obstacle is more dramatic in psychiatric disorders since their diagnosis primarily depend on the symptoms and signs of the patients. In this paper, we reviewed this rapidly advancing field by discussing definition of extracellular vesicles, their biogenesis and potential use as clinical biomarkers. Then we focused on their potential use in psychiatric disorders in the context of diagnosis and treatment of these disorders. Finally, we tried to combine the RDoC (Research Domain Criteria) with the use of extracellular vesicles in psychiatry research and practice. This review may offer new insights in both basic and translational research focusing on psychiatric disorders.
\end{abstract}

Psychiatry Investig 2018;15(11):1011-1018

Key Words Extracellular vesicle, Depression, Bipolar disorder, Schizophrenia, Research domain criteria.

\section{INTRODUCTION}

The cells of multicellular organisms developed various ways of communication for maintaining homeostasis and response to their inner and outer environment. ${ }^{1}$ In addition to endocrine, paracrine, juxtacrine and autocrine signaling, the cells send signals via extracellular membrane bound structures, i.e. extracellular vesicles, and can be classified depending on their size, molecular composition and synthesis pathways. ${ }^{2}$ The cells within central nervous system uses these extracellular vesicles for long distance communication, which allows nucleic acid (both DNA and different types RNA), protein and special lipid domain transfer between the cells. ${ }^{3}$

The extracellular vesicles can be classified as exosomes, which has a diameter ranging from $30 \mathrm{~nm}$ to $100 \mathrm{~nm}$; microvesicles with diameter between $100-1000 \mathrm{~nm}$; apoptotic bodies, which are released from dying cells via apoptotic process, and has a diameter between $800-5000 \mathrm{~nm}$, and large on-

Received: July 27, 2018 Revised: September 6, 2018

Accepted: September 17, 2018

$\triangle$ Correspondence: Can Ilgin, MD, PhD

Department of Public Health, Marmara University School of Medicine, Basibuyuk Mahallesi, Maltepe Basibuyuk Yolu Sokak, Maltepe-İstanbul 34854, Turkey

Tel: +902164212222, Fax: +902164144731, E-mail: can.ilgin@hotmail.com

(a) This is an Open Access article distributed under the terms of the Creative Commons Attribution Non-Commercial License (http://creativecommons.org/licenses/bync/4.0) which permits unrestricted non-commercial use, distribution, and reproduction in any medium, provided the original work is properly cited. cosomes, which are released from neoplastic cells and has a diameter larger than $1 \mu \mathrm{m}$ and can be large as $10 \mu \mathrm{m} .{ }^{4,5}$

\section{BIOGENESIS OF EXTRACELLULAR VESICLES}

The exosomes are generated via multivesicular bodies (MVB) which are derived from early endosomes. Early endosomes contain lipid rafts with specific proteins e.g. tetraspanins, adhesion, fusion, and receptor transport proteins. With the intraluminal vesicle (ILV) formation, these molecules are incorporated into the exosomal membrane. ${ }^{6}$ ESCRT, TSG101, Alix proteins are responsible for the sorting of exosomes and after packaging, exosomes expelled to the extracellular domain via a fusion process led by Rab GTPases (Figure 1).?

Microvesicles are the generated through the budding of plasma membrane, which is regulated through translocation of phospholipids by floppase/flippase enzymes, organization of cytoskeleton under plasma membrane with ADP-ribosylation factor 6 (ARF6), Phospholipase D (PLD), Extracellularsignal regulated kinase (ERK) and Myosin light-chain kinase (MLCK) enzymes. ${ }^{8}$ The molecules participating into extracellular vesicle biogenesis process are candidates for EV markers, which useful in detection and isolation of EVs (Figure 2). ${ }^{9}$ 


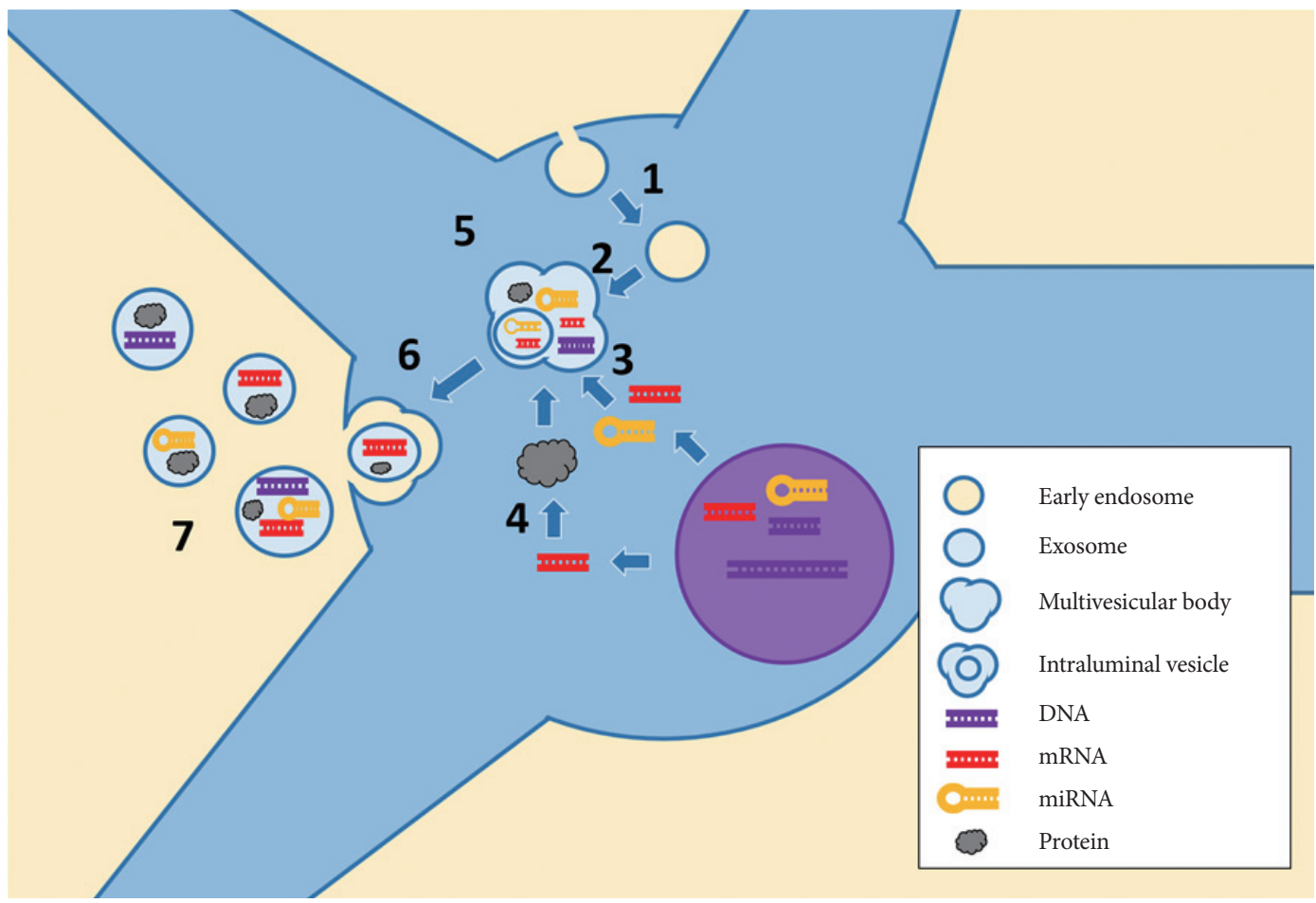

Figure 1. Exosomes Biogenesis The biogenesis of exosomes occurs within the cells through a multistep process. In this figure, the exosome formation is simplified to emphasize the important steps in this cellular process. 1. The exosome formation starts with the inward budding of plasma membranes into the cytoplasm (endocytosis), which leads to the early endosome formation. 2. Early endosomes accumulate and fuse to form multivesicular bodies (MVB). 3. Nucleic acids (mRNA, miRNA, other RNA molecules and fragments of DNA) from the nucleus are transported into the MVB, and accumulate within this membrane bound structure. 4. Some mRNAs translated to protein structures through ribosomes and these proteins are transported into the MVB. 5. The outer membrane of MVB form another internal compartment by budding into this structure. This vesicle inside of MVB is called intraluminal vesicle (ILV). Proteins and nucleic acids are sorted into the ILVs through molecular sorting mechanisms composed of proteins like ESCRT (Endosomal sorting complexes required for transport), TSG 101 (Tumor susceptibility gene), and ALIX (ALG-2-interacting protein X). 6. Exosomes expelled from the cell via exocytosis, where MVB docks with plasma membrane via Rab proteins, and unloads its exosome cargo. The contents of exosomes are diverse, which are composed of various proteins and nucleic acids. 7. Exosomes are secreted to the extracellular compartment. These membrane bound structures can travel through blood and lymphatic vessels, cerebrospinal fluid (CSF), saliva and other secretions of the body. Exosomes possess adhesion and receptor proteins on their surfaces, which allows the exosomes to bind to their specific targets.

\section{ISOLATION OF EXTRACELLULAR VESICLES}

EVs can be isolated from serum, saliva, cerebrospinal fluid (CSF), urine, breast milk, synovial fluid, effusions, semen and cell cultures. ${ }^{10}$ The isolation of EVs requires either different centrifuge steps up to an ultracentrifuge with $100,000 \mathrm{~g}$ and/ or mechanical filters, which are specifically designed according to diameter preferred EV subtype. ${ }^{11}$ The EV purification and subpopulation selection can be performed with immunocapture methods. ${ }^{12}$ The isolated EVs can be imaged with transmission and/or scanning electron microscopes. Transmission electron microscopy can be combined with immunogold method to show a specific protein within or on the $\mathrm{EV}^{13}$ The light microscopes with capability of super-resolution can be recruited for the imaging of EVs, particularly for exosomes, which has a diameter smaller than $100 \mathrm{~nm} .{ }^{14}$ The isolated EVs can be analyzed for their content of proteins, nucleic ac- ids, lipid and carbohydrates. ${ }^{15}$

\section{EXTRACELLULAR VESICLES ORIGINATING FROM CENTRAL NERVOUS SYSTEM}

Since CNS is sealed by bones, connective tissues and bloodbrain barriers, also contains vital centers for cognition, sensory, motor and autonomic functions, the indications for taking direct biopsies for diagnostic purposes is limited only to some life threating conditions like infection/abscess or brain tumors. ${ }^{16}$ Many central nervous system pathologies are diagnosed with imaging modalities, including computed tomography $(\mathrm{CT})$ and magnetic resonance imaging (MRI). ${ }^{17}$ Through these imaging modalities are capable of noninvasively monitoring the organs within CNS, generally they don't any functional information regarding cellular processes. ${ }^{18}$ The functional imaging modalities like functional MRI (fMRI) or 


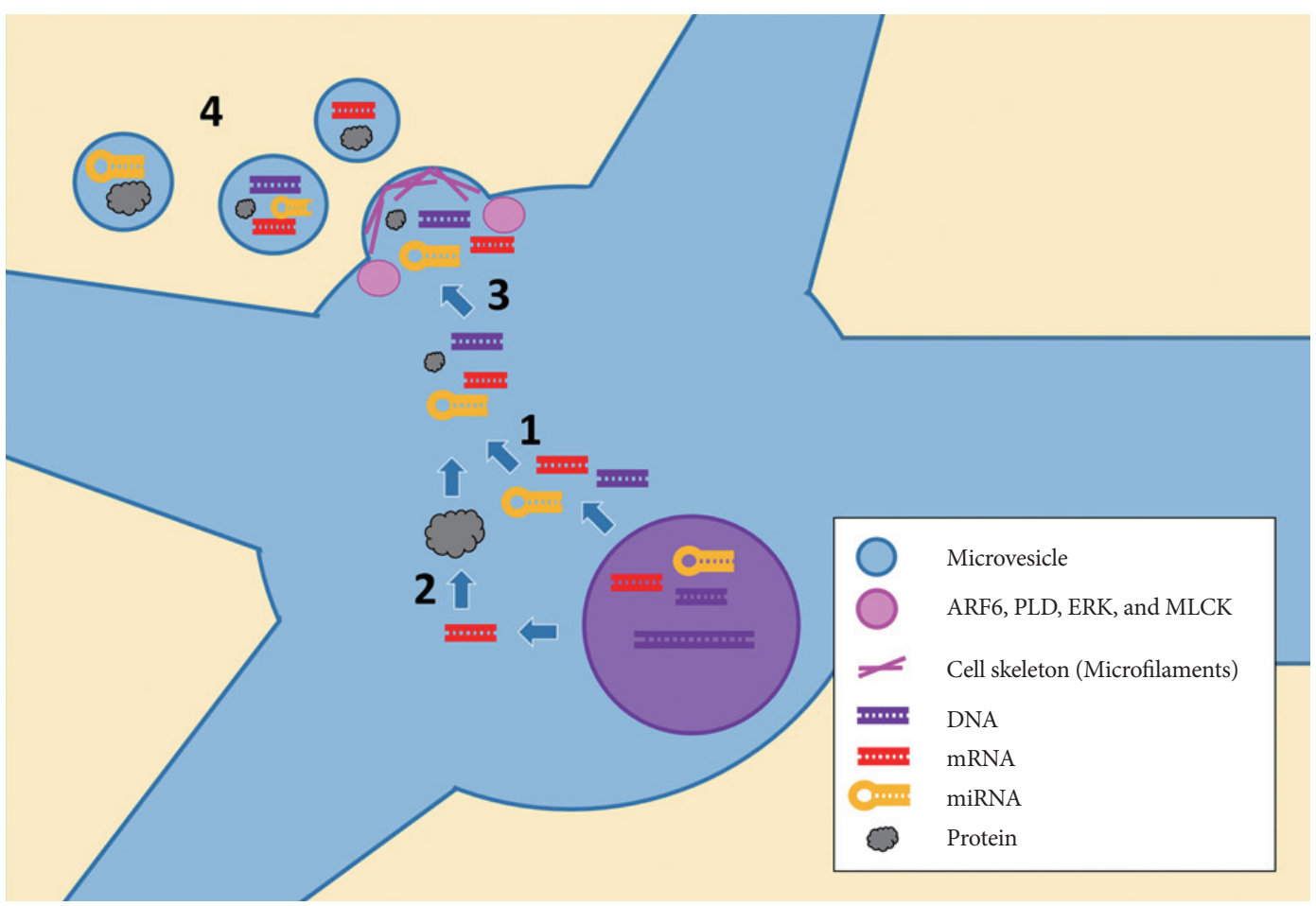

Figure 2. Microvesicle biogenesis The biogenesis of microvesicles occurs mainly on plasma membrane and adjacent cytoplasmic structures (including microfilaments and cytoplasm), through a multistep process. In this figure, the microvesicle formation is simplified to emphasize the important steps in this cellular process. 1. Nucleic acids from the nucleus are processed and transported to the cytoplasm, where they will be transported to the cytoplasmic domain. 2. Some mRNAs are translated to the proteins, which are transported to the cytoplasmic domain under the plasma membrane for packaging process. 3. Nucleic acids and proteins are transported to the plasma membrane domain, where the microvesicle formation will take place. The molecular cargo is sorted into the microvesicles through the molecular sorting machinery (e.g. ESCRT system). Enzymes and proteins e.g. ARF6 (ADP-ribosylation factor 6), PLD (Phospholipase D), ERK (Extracellular-signal regulated kinases) and MLCK (Myosin light-chain kinase) are responsible for the cell skeleton (mainly microfilament) organization. The floppase/flippase enzymes regulate the lipid domain content during the microvesicle formation process. 4. Microvesicles are secreted to the extracellular compartment. These membrane bound structures can travel through blood and lymphatic vessels, cerebrospinal fluid (CSF), saliva and other secretions of the body. Microvesicles possess adhesion and receptor proteins on their surfaces, which allows the microvesicles to bind to their specific targets.

positron emission tomography (PET) shows the metabolic activity in brain, however these techniques still suffers the resolution problem, since they can't monitor individual neuronal activity. ${ }^{19}$ The best technique for gaining biochemical, cellular or microbiological information from CNS is obtaining CSF via lumbar puncture. ${ }^{20} \mathrm{EVs}$ can be collected form CSF and provide valuable information regarding the functional status of neurons and glial cells within the CNS. ${ }^{21}$ However, lumbar puncture is still an invasive method with its possible complications e.g. hemorrhage, spinal cord/nerve root injury, even cerebral hernia. ${ }^{22}$ In addition, the content of CSF is modified through the components of CSF- brain barrier, i.e. choroid plexus/ ependymal cells, neural and glial processes. ${ }^{23}$ Exosomes from CNS carry molecules originating from the inner compartment, including ILV and EBV machinery, of their source cells (neurons, glial cells). In addition, these vesicles carry the inside information and messages from the CNS cells, which reside in a sealed biological compartment. It can be hypothesized, that the information within the exo- somes is changed minimally during their journey from CNS to the peripheral blood. Thus, exosomes might contain more proper information regarding cellular/metabolic status within CNS when they are compared to CSF.

An alternative and relatively noninvasive way to obtain information from CNS is to collect EVs which are shed from blood brain barrier. ${ }^{24} \mathrm{EV}$ s from blood brain barrier contains EVs from both CNS and blood brain barrier components, i.e. endothelial cells and astrocytes. ${ }^{25}$ The EVs from neurons and glial cells can cross blood brain barrier via transcytosis process. ${ }^{26}$ The main limitations for obtaining EVs from peripheral blood sample are isolation of blood brain barrier derived EVs from peripheral EVs and the determination of origins of EVs derived from blood brain barrier. The areas where sealing of blood brain barrier is relatively weak (e.g. circumventricular organs) might be comparatively more direct source for exosome originating from $\mathrm{CNS} .{ }^{27} \mathrm{In}$ addition, EVs with neuronal origins can be isolated from peripheral blood samples through various surface markers like NCAM or L1CAM. ${ }^{28}$ 
Exosomes with neural origins also carry GLP-anchored prion protein and Glutamate receptor GluR2/3 subunits. Since these proteins are shown in neural cell culture as neural exosomal markers, more research is needed for their use for peripheral blood neural exosome isolation. ${ }^{29,30}$ The proteomic analysis of exosomes derived from microglia culture showed the expression of CD13 and lactate transporter MCT- $1 .{ }^{31}$ The exosomes with oligodendroglia origin has myelin components, like myelin basic protein or myelin oligodendrocyte glycoprotein. ${ }^{32}$ Another approach for the isolation of CNS derived exosomes is the use lipidomic and proteomic profiles of these exosomes. The lipid composition of exosomes (phosphatidylserine, sphingomyelin, lyso-phosphatidylcholine, lysophosphatidylethanolamine and other families), provide information regarding the origin of exosomes. ${ }^{33}$

\section{PSYCHIATRIC DISORDERS AND EXTRACELLULAR VESICLES}

The isolation and biochemical analysis of EVs in psychiatric disorders can offer wide diagnostic and therapeutic opportunities, in addition to provision of valuable information regarding pathophysiology of these diseases. ${ }^{34}$ In current clinical practice, imaging of CNS is not in routine use for the differential diagnosis of psychiatric diseases. ${ }^{35}$ The blood tests are usually recruited for monitoring drug levels, ruling out endocrine diseases, micronutrient deficiencies or organ failures which might affect the mental status of patients. ${ }^{36,37}$ However, blood tests are not capable to demonstrate the central nervous system biochemical and cellular properties. The information regarding the level of neurotransmitters, neural metabolic status, epigenetic and/or genetic alterations in neurons would provide valuable information for diagnosis and individualization of treatment of psychiatric disorders.

Nevertheless, the previous studies focusing on EVs in psychiatric disorders are predominantly executed in postmortem brain tissues of the patients. ${ }^{38-41}$ The use of EVs extracted from peripheral blood samples for diagnostic purposes requires further basic research and clinical studies carried on patients diagnosed with psychiatric disorders. However, these preceding ex-vivo (postmortem) studies might guide the researchers to find potential targets to evaluate in patient population in order to develop biomarkers for diagnostic and/or therapeutic purposes.

In this part of the review, we will focus on some important psychiatric disorders, in which the use of EVs might be beneficial in terms of basic research, diagnosis (including secondary prevention of psychiatric disorders) and treatment. We decided to review EVs in depression, bipolar disorder, schizophrenia, and Alzheimer's disease in the context of Research
Domain Criteria (RDoC), since the constructs of these criteria attempt to reflect the etiology of psychiatric diseases in different dimension, extending from the molecules to the neural circuitries and anatomical structures. ${ }^{42}$ Although the previous studies on EV in psychiatric disorders do not cover all aspects of RDoC criteria, understanding of EV characteristics in relation to a specific $\mathrm{RDoC}$ construct is still important to clarify the pathogenesis and finding appropriate biomarkers for these disorders.

\section{Depression and extracellular vesicles}

Depression is a major public health problem linked with decreased functionality and may result in mortality. ${ }^{43}$ The pathophysiology of depression is linked with increased activity in hypothalamic-pituitary-adrenal axis, hypo-connectivity in frontoparietal network, structural changes in brain, alterations in neural and glial cells, abnormal brain activity and neurotransmitter functions (involving GABA, glutamate, serotonin, epinephrine and norepinephrine) ${ }^{44,45}$

According to an analysis using RDoC Negative Valence Systems matrix, depression is associated with disruptions in certain neural circuits, e.g. cortico-limbic circuitry, some key genes involved in neurotransmitter metabolism (MAOA, COMT, DATI, 5HTTR, 5HTRs), glucocorticoid receptor downregulation, $\mathrm{CRH}$, sex steroid, oxytocin, vasopressin upregulation, dysregulations in autonomic nervous system, hypothalamic-pituitary-adrenal axis, neuro-inflammation and extended reactivity and behavioral changes (e.g. withdrawal, sadness). ${ }^{46}$ RDoC Negative Valence Systems matrix demonstrates different etiological pathways, which might lead to signs and symptoms of depression.

One of the components of Negative Valence Systems matrix, neuro-inflammation, occurs in depression patients within CNS and associated with the activation of microglial cells. ${ }^{47}$ Neuro-inflammation is related to a decreased neurogenesis and increase in glutamate excitation toxicity. ${ }^{48,49}$ The neuroinflammatory microenvironment seen in depression causes morphological and functional changes in microglia via LPS molecule. The activated microglia cells secrete higher amounts of EVs, which are loaded with proinflammatory cytokines, e.g. IL-1 $\beta$, caspase-1 etc., in addition to secretion of proinflammatory cytokines (IL-6, TNF- $\alpha$ ) via exocytosis. MAPK, JNK1/2, P38 and NFKB pathways are responsible for this functional change within ILV/MVB exosome biogenesis. The expression of various miRNA molecules e.g. miR-1202, Let7a, mir124, which have crucial functions in neurogenesis, neurotransmission (dopaminergic, GABAergic, serotonergic pathways), and ion channel regulation, are altered in several areas (prefrontal cortex, frontal cortex) of CNS. ${ }^{39}$ Levels of these miRNAs, like miR-132 (upregulation), miR-1202 (downregula- 
tion), are altered in the peripheral blood samples of patients, which are mainly carried via EVs through bloodstream and have diagnostic potential. ${ }^{50}$

\section{Bipolar disorders and extracellular vesicles}

The bipolar disorders are characterized with mania or hypomania, which are accompanied with major depressive attacks. Bipolar disorder pathophysiology is explained though anatomical and functional changes in brain architecture (including altered connectivity in anterior cingulate cortex, prefrontal cortex and amygdala), and neuro-inflammation. ${ }^{51}$

According to the $\mathrm{RDoC}$ positive valance system matrix, bipolar disorders are linked with abnormally elevated reward activation, and excess approach motivation, which leads to approach related hypomanic/manic symptoms. ${ }^{52,53}$ Bipolar disorder is also linked with $\mathrm{RDoC}$ arousal systems matrix. ${ }^{54}$ Anterior limbic system, amygdala and orbitofrontal cortex are associated with reward valuation, reward related decision, in addition to their association..$^{55}$ The dopaminergic neurons in midbrain (including basal ganglia) are responsible for approach motivation. ${ }^{56}$

The studies carried on EVs obtained from patients with bipolar disorder show concordance with $\mathrm{RDoC}$ matrices in terms of anatomical locations, where pathological processes take place. The brain specific miRNA-134 is responsible for the formation of dendritic spines and synapses and can be obtained via blood samples and used for monitoring and treatment of mania episodes in bipolar disorder. ${ }^{57}$ In addition, miR-29c expression is increased in extracellular vesicles extracted from prefrontal cortex samples of patients with bipolar disorder. miR-29c is linked with neural development and signaling, and a target for lithium treatment. ${ }^{38}$ The analysis of EVs from anterior cingulate cortex of bipolar disorder patients showed increased miR-149 and miR-29c expressions. The increased miR-149 expression originates from the glial cells and inhibits glial cell proliferation. ${ }^{40}$ Exosomes originating from astrocytes within niche of neurogenesis might transfer the stress signals from peripheral blood (e.g. corticosteroids, cytokines) and disturb neurogenesis in depression and bipolar disorder. ${ }^{58}$ Exosome characteristics could be a marker for different stages of bipolar disease and prognosis.

\section{Schizophrenia and extracellular vesicles}

Schizophrenia is characterized by reoccurring or chronic psychosis along with other positive and negative (involving mood and cognition deficits) symptoms. ${ }^{59}$ Pathophysiology of schizophrenia is associated with abnormal neurotransmission (including dopamine, serotonin, glutamate and many other neurotransmitters), genetic predisposition (including synaptogenesis, neurotransmitters, and immune system), in- trauterine and early childhood exposures, autoimmune disorders and substance use. Schizophrenia is linked excess dopaminergic activity in mesolimbic tract, decreased dopamine in prefrontal cortex, decrease in NMDA glutamate receptor (mesocortical dopamine neurons) and GABAergic inhibitory activities. $^{60-62}$

According to the RDoC positive valance system matrix, schizophrenia is linked with altered reward valuation/prediction linked with prefrontal/striatal activation, reduced reward learning and action selection. The impairment in explicit positive reinforced learning can be explained with increased dopamine in basal ganglia and reduced dorsolateral prefrontal cortex activity, which also explains the impairment the action towards valuated outcomes. ${ }^{63}$ The auditory hallucinations in schizophrenia are linked with cognition, social processes and negative valance constructs (including sustained and acute threat). ${ }^{64}$

The postmortem prefrontal cortex samples of schizophrenia patients have increased amounts of exosomal miR-497, when it's compared to control patients. ${ }^{38,65}$ In addition to miRNAs, EVs can transport misfolded proteins or neurotransmitters, which might play a part in the pathogenesis in schizophrenia. ${ }^{41}$ Since the exosomes are capable to carry large molecules like brain-derived neurotrophic factor or platelet-derived growth factor receptor, it can hypothesized that extracellular vesicles might also carry neurotransmitter receptors on their membranes. ${ }^{66,67}$ Glutamatergic and dopaminergic receptors on extracellular vesicles might provide valuable information regarding the neural receptor expression status and help to finetune the treatment.

\section{Alzheimer's disease and extracellular vesicles}

Alzheimer's disease (AD) is characterized with increasing decline of cognitive functions as a result of neural degeneration, especially in older individuals. The most noticeable feature of $\mathrm{AD}$ is the progressive memory loss but it's commonly accompanied by other cognitive deficits e.g. behavioral, and psychological signs. ${ }^{68}$

The pathophysiology of $\mathrm{AD}$ is characterized by accumulations of Tau and $\beta$-amyloid proteins in intra- and extracellular compartments, respectively. ${ }^{69}$ The etiology of $\mathrm{AD}$ can be explained with genetic (e.g. amyloid precursor protein APP, presenillin, and some ApoE alleles), metabolic (e.g. diabetes mellitus, dyslipidemia, obesity), vascular (e.g. cerebral atherosclerosis, hypertension), neuro-inflammatory factors (e.g. trauma, free radicals)..$^{70-72}$ The neuropsychiatric symptoms in $\mathrm{AD}$, like agitation, depression or delusions, can be linked with $\mathrm{RDoC}$ cognitive systems domain, however there is significant overlapping between different $\mathrm{RDoC}$ constructs by explaining these signs and symptoms. ${ }^{73}$ 
The previous studies revealed that some fraction of $\beta$-amyloid protein secretion to extracellular compartment occurs through exosomes. Amyloid precursor protein (APP) is cleaved into $\beta$-amyloid proteins and fragments of $\beta$-carboxyl-terminal (CTFs) via secretase enzymes ( $\beta$ and $\gamma$, which are involved in $\mathrm{AD}$ pathogenesis) within exosomes. This cleavage process begins in endosomes and multivesicular bodies within the intracellular compartment, and continues in the exosomes in extracellular domain. The $\beta$ and $\gamma$ cleavage products of APP leads the formation of $\beta$-amyloid plaques in extracellular compartment of CNS. ${ }^{74,75}$ The $\beta$-amyloid formation also increases the neuro-inflammation within the CNS. ${ }^{76}$

Additionally, exosomes are also responsible for spreading of the hyper-phosphorylated Tau proteins in AD. Thus, these neurofibrillary tangles may contaminate the healthy neurons via EVs and interfere with the metabolism and intracellular neurotransmitter transport system of these cells. ${ }^{77}$

Nevertheless, it is also demonstrated that the neural exosomes are responsible for clearance of $\beta$-amyloid plaques via microglia within CNS. ${ }^{78}$ This shows the both detrimental and beneficial effects of exosomes in the pathogenesis of $\mathrm{AD}$.

Along with the proteins, miRNAs are also released within exosomes and play significant roles in $\mathrm{AD}$ pathogenesis. The alterations in exosomal miRNA expressions in $\mathrm{AD}$ can be detected in CSF and/or peripheral blood samples. The role of miRNAs in neurodegeneration is multifaceted, while some miRNA molecules (like miRNA-219, miR-124a) favor myelination and signaling, others (like miR-193b, let-7) accelerate neural degeneration through increasing neuro-inflammation and amyloid plaque formation. ${ }^{79}$

\section{CONCLUSION}

The $\mathrm{RDoC}$ constructs provide valuable information for choosing candidates for biomarker molecules for both diagnostic and therapeutic purposes in psychiatric disorders. By using methods of bioinformatics, these potential biomarkers can be extracted from RDoC domain matrices. Extracellular vesicles provide a chance for measuring different classes of biomarkers (e.g. miRNA, proteins and lipids) simultaneously, which might increase the predictive values of testing and help to overcome the difficulties of working with a diagnostic modality relying on a single class of molecule. Using multiple markers on extracellular vesicles carry a huge potential for improving of understanding and managing of psychiatric disorders.

\section{REFERENCES}

1. Conigliaro A, Fontana S, Raimondo S, Alessandro R. Exosomes: nanocarriers of biological messages. Adv Exp Med Biol 2017;998:23-43.
2. Paolicelli RC, Bergamini G, Rajendran L. Cell-to-cell communication by extracellular vesicles: focus on microglia. Neuroscience 2018 [Epub ahead of print].

3. Tietje A, Maron KN, Wei Y, Feliciano DM. Cerebrospinal fluid extracellular vesicles undergo age dependent declines and contain known and novel non-coding RNAs. PLoS ONE 2014;9:e113116.

4. Crescitelli R, Lässer C, Szabó TG, Kittel A, Eldh M, Dianzani I, et al. Distinct RNA profiles in subpopulations of extracellular vesicles: apoptotic bodies, microvesicles and exosomes. J Extracell Vesicles 2013;2.

5. Minciacchi VR, Freeman MR, Di Vizio D. Extracellular vesicles in cancer: exosomes, microvesicles and the emerging role of large oncosomes. Semin Cell Dev Biol 2015;40:41-51.

6. Huotari J, Helenius A. Endosome maturation. EMBO J 2011;30:34813500 .

7. Ha D, Yang N, Nadithe V. Exosomes as therapeutic drug carriers and delivery vehicles across biological membranes: current perspectives and future challenges. Acta Pharm Sin B 2016;6:287-296.

8. Akers JC, Gonda D, Kim R, Carter BS, Chen CC. Biogenesis of extracellular vesicles (EV): exosomes, microvesicles, retrovirus-like vesicles, and apoptotic bodies. J Neurooncol 2013;113:1-11.

9. Rajagopal C, Harikumar KB. The origin and functions of exosomes in cancer. Front Oncol 2018;8:66.

10. Xu W, Yang Z, Lu N. From pathogenesis to clinical application: insights into exosomes as transfer vectors in cancer. J Exp Clin Cancer Res 2016;35:156.

11. Lobb RJ, Becker M, Wen Wen S, Wong CSF, Wiegmans AP, Leimgruber A, et al. Optimized exosome isolation protocol for cell culture supernatant and human plasma. J Extracell Vesicles 2015;4:27031.

12. Theodoraki MN, Hoffmann TK, Whiteside TL. Separation of plasmaderived exosomes into $\mathrm{CD} 3((+))$ and $\mathrm{CD} 3((-))$ fractions allows for association of immune cell and tumour cell markers with disease activity in HNSCC patients. Clin Exp Immunol 2018;192:271-283.

13. Carpintero-Fernández P, Fafián-Labora J, O’Loghlen A. Technical advances to study extracellular vesicles. Front Mol Biosci 2017;4:79.

14. Banfer S, Schneider D, Dewes J, Strauss MT, Freibert SA, Heimerl T, et al. Molecular mechanism to recruit galectin-3 into multivesicular bodies for polarized exosomal secretion. Proc Natl Acad Sci U S A 2018; 115:E4396-E4405.

15. Merchant ML, Rood IM, Deegens JKJ, Klein JB. Isolation and characterization of urinary extracellular vesicles: implications for biomarker discovery. Nat Rev Nephrol 2017;13:731-749.

16. Bekelis K, Radwan TA, Desai A, Roberts DW. Frameless robotically targeted stereotactic brain biopsy: feasibility, diagnostic yield, and safety. J Neurosurg 2012;116:1002-1006.

17. Albrecht DS, Granziera C, Hooker JM, Loggia ML. In Vivo Imaging of Human Neuroinflammation. ACS Chem Neurosci 2016;7:470-483.

18. Fabi A, Vidiri A. Defining the endpoints: how to measure the efficacy of drugs that are active against central nervous system metastases. Transl Lung Cancer Res 2016;5:637-646.

19. Ferezou I MF, Petersen CCH. Imaging the Brain in Action: Real-Time Voltage- Sensitive Dye Imaging of Sensorimotor Cortex of Awake Behaving Mice. In: RD F, Editor. In Vivo Optical Imaging of Brain Function 2nd Edition. Florida: CRC Press/Taylor \& Francis, 2009, p.171192.

20. Arboix A, Obach V, Sánchez MJ, Massons J. Complementary examinations other than neuroimaging and neurosonology in acute stroke. World J Clini Cases 2017;5:191-202.

21. Borroto-Escuela DO, Agnati LF, Bechter K, Jansson A, Tarakanov AO, Fuxe K. The role of transmitter diffusion and flow versus extracellular vesicles in volume transmission in the brain neural-glial networks. Philos Trans R Soc Lond B Biol Sci 2015;370.

22. Doherty CM, Forbes RB. Diagnostic lumbar puncture. Ulster Med J 2014;83:93-102.

23. Di Terlizzi R, Platt S. The function, composition and analysis of cerebrospinal fluid in companion animals: part I-function and composi- 
tion. Vet J 2006; 172:422-431.

24. Narahari A, Hussain M, Sreeram V. MicroRNAs as biomarkers for psychiatric conditions: a review of current research. Innov Clin Neurosci 2017;14:53-55.

25. Andras IE, Toborek M. Extracellular vesicles of the blood-brain barrier. Tissue Barriers 2016;4:e1131804.

26. Matsumoto J, Stewart T, Banks WA, Zhang J. The transport mechanism of extracellular vesicles at the blood-brain barrier. Curr Pharm Des 2017;23:6206-6014.

27. Smalheiser NR. Do neural cells communicate with endothelial cells via secretory exosomes and microvesicles? Cardiovasc Psychiatry Neurol 2009;2009:383086.

28. Mustapic M, Eitan E, Werner JK, Berkowitz ST, Lazaropoulos MP, Tran J, et al. Plasma extracellular vesicles enriched for neuronal origin: a potential window into brain pathologic processes. Front Neurosci 2017;11:278.

29. Fauré J, Lachenal G, Court M, Hirrlinger J, Chatellard-Causse C, Blot $\mathrm{B}$, et al. Exosomes are released by cultured cortical neurones. $\mathrm{Mol} \mathrm{Cel}$ Neurosci 2006;31:642-648.

30. Kanninen KM, Bister N, Koistinaho J, Malm T. Exosomes as new diagnostic tools in CNS diseases. Biochim Biophys Acta 2016;1862:403-410.

31. Potolicchio I, Carven GJ, Xu X, Stipp C, Riese RJ, Stern LJ, et al. Proteomic analysis of microglia-derived exosomes: metabolic role of the aminopeptidase CD13 in neuropeptide catabolism. J Immunol 2005; 175:2237-2243.

32. Kramer-Albers EM, Bretz N, Tenzer S, Winterstein C, Mobius W, Berger $\mathrm{H}$, et al. Oligodendrocytes secrete exosomes containing major myelin and stress-protective proteins: Trophic support for axons? Proteomics Clin Appl 2007;1:1446-1461.

33. Gallart-Palau X, Serra A, Sze SK. Enrichment of extracellular vesicles from tissues of the central nervous system by PROSPR. Mol Neurodegener 2016;11:41.

34. Raghavan V. Role of exosomes in psychiatric disorders. Asian J Psychiatr 2017;28:78-79.

35. Masdeu JC. Neuroimaging in psychiatric disorders. Neurotherapeutics 2011;8:93-102.

36. The Lancet Psychiatry. Blood biomarkers in psychiatry. Lancet Psychiatry 2016;3:693.

37. Hiemke C. Clinical utility of drug measurement and pharmacokinetics: therapeutic drug monitoring in psychiatry. Eur J Clin Pharmacol 2008;64:159-166.

38. Banigan MG, Kao PF, Kozubek JA, Winslow AR, Medina J, Costa J, et al. Differential expression of exosomal microRNAs in prefrontal cortices of schizophrenia and bipolar disorder patients. PLoS One 2013;8: e48814.

39. Brites D, Fernandes A. Neuroinflammation and depression: microglia activation, extracellular microvesicles and microRNA dysregulation. Front Cell Neurosci 2015;9:476.

40. Choi JL, Kao PF, Itriago E, Zhan Y, Kozubek JA, Hoss AG, et al. miR149 and miR-29c as candidates for bipolar disorder biomarkers. Am J Med Genet B Neuropsychiatr Genet 2017;174:315-323.

41. Pegtel DM, Peferoen L, Amor S. Extracellular vesicles as modulators of cell-to-cell communication in the healthy and diseased brain. Philos Trans R Soc Lond B Biol Sci 2014;369.

42. Sawa A. Multifaceted Translational Approach to Major Mental Illness. In: Nakao K, Minato N, Uemoto S, Editors. Innovative Medicine: Basic Research and Development. Tokyo: Springer, 2015, p.157-163.

43. Jacob KS. Depression: a major public health problem in need of a multi-sectoral response. Indian J Med Res2012;136:537-539.

44. Kaiser RH, Andrews-Hanna JR, Wager TD, Pizzagalli DA. Large-scale network dysfunction in major depressive disorder: a meta-analysis of resting-state functional connectivity. JAMA Psychiatry 2015;72:603611.

45. Zhang HF, Mellor D, Peng DH. Neuroimaging genomic studies in major depressive disorder: a systematic review. CNS Neurosci Ther 2018
[Epub ahead of print].

46. Woody ML, Gibb BE. Integrating NIMH Research Domain Criteria (RDoC) into depression research. Curr Opin Psychol 2015;4:6-12.

47. Singhal G, Baune BT. Microglia: an Interface between the loss of neuroplasticity and depression. Front Cell Neurosci 2017;11:270.

48. Kim YK, Na KS. Role of glutamate receptors and glial cells in the pathophysiology of treatment-resistant depression. Prog Neuropsychopharmacol Biol Psychiatry 2016;70:117-126.

49. Kim YK, Na KS, Myint AM, Leonard BE. The role of pro-inflammatory cytokines in neuroinflammation, neurogenesis and the neuroendocrine system in major depression. Prog Neuropsychopharmacol Biol Psychiatry 2016;64:277-284.

50. Lopez JP, Kos A, Turecki G. Major depression and its treatment: microRNAs as peripheral biomarkers of diagnosis and treatment response. Curr Opin Psychiatry 2018;31:7-16.

51. Woelfer M, Kasties V, Kahlfuss S, Walter M. The role of depressive subtypes within the neuroinflammation hypothesis of major depressive disorder. Neuroscience 2018 [Epub ahead of print].

52. Ogasawara K, Nakamura Y, Kimura H, Aleksic B, Ozaki N. Issues on the diagnosis and etiopathogenesis of mood disorders: reconsidering DSM-5. J Neural Transm (Vienna) 2018;125:211-222.

53. Nusslock R, Alloy LB. Reward processing and mood-related symptoms: An RDoC and translational neuroscience perspective. J Affect Disord 2017;216:3-16.

54. Cosgrove VE, Kelsoe JR, Suppes T. Toward a valid animal model of bipolar disorder: how the research domain criteria help bridge the clinical-basic science divide. Biol Psychiatry 2016;79:62-70.

55. Rolls ET. Limbic systems for emotion and for memory, but no single limbic system. Cortex 2015;62:119-157.

56. Ikemoto S, Yang C, Tan A. Basal ganglia circuit loops, dopamine and motivation: a review and enquiry. Behavioural brain research. Behav Brain Res 2015;290:17-31.

57. Rong H, Liu TB, Yang KJ, Yang HC, Wu DH, Liao CP, et al. MicroRNA-134 plasma levels before and after treatment for bipolar mania. J Psychiatr Res 2011;45:92-95.

58. Luarte A, Cisternas P, Caviedes A, Batiz LF, Lafourcade C, Wyneken U, et al. Astrocytes at the hub of the stress response: potential modulation of neurogenesis by miRNAs in astrocyte-derived exosomes. Stem Cells Int 2017;2017:1719050.

59. Picchioni MM, Murray RM. Schizophrenia. BMJ 2007;335:91-95.

60. Debnath M, Venkatasubramanian G, Berk M. Fetal programming of schizophrenia: select mechanisms. Neurosci Biobehav Rev 2015;49:90104.

61. Patel KR, Cherian J, Gohil K, Atkinson D. Schizophrenia: overview and treatment options. P T 2014;39:638-645.

62. Faludi G, Mirnics K. Synaptic changes in the brain of subjects with schizophrenia. Int J Dev Neurosci 2011;29:305-309.

63. Barch DM, Pagliaccio D, Luking K. Mechanisms Underlying Motivational Deficits in Psychopathology: Similarities and Differences in Depression and Schizophrenia. In: Simpson EH, Balsam PD, Editors. Behavioral Neuroscience of Motivation. Cham: Springer International Publishing, 2016, p. 411-449.

64. Ford JM, Morris SE, Hoffman RE, Sommer I, Waters F, McCarthyJones S, et al. Studying Hallucinations Within the NIMH RDoC Framework. Schizophr Bull 2014;40(Suppl 4):S295-S304.

65. Nascimento Juliana M, Garcia S, Saia-Cereda Verônica M, Santana Aline G, Brandao-Teles C, Zuccoli Giuliana S, et al. Proteomics and molecular tools for unveiling missing links in the biochemical understanding of schizophrenia. Proteomics Clin Appl 2016;10:1148-1158.

66. Suire CN, Eitan E, Shaffer NC, Tian Q, Studenski S, Mattson MP, et al. Walking speed decline in older adults is associated with elevated proBDNF in plasma extracellular vesicles. Exp Gerontol 2017;98:209-216.

67. Pituch KC, Moyano AL, Lopez-Rosas A, Marottoli FM, Li G, Hu C, et al. Dysfunction of platelet-derived growth factor receptor alpha (PDGFRalpha) represses the production of oligodendrocytes from arylsulfa- 
tase A-deficient multipotential neural precursor cells. J Biol Chem 2015;290:7040-7053.

68. Kumar K, Kumar A, Keegan RM, Deshmukh R. Recent advances in the neurobiology and neuropharmacology of Alzheimer's disease. Biomed Pharmacother 2018;98:297-307.

69. Weller J, Budson A. Current understanding of Alzheimer's disease diagnosis and treatment. F1000Res 2018;7. pii: F1000 Faculty Rev-1161.

70. Craft S. The role of metabolic disorders in Alzheimer's disease and vascular dementia: two roads converged? Arch Neurol 2009;66:300305.

71. Giri M, Zhang M, Lu Y. Genes associated with Alzheimer's disease: an overview and current status. Clin Interv Aging 2016;11:665-681.

72. Guedes JR, Lao T, Cardoso AL, El Khoury J. Roles of microglial and monocyte chemokines and their receptors in regulating Alzheimer's disease-associated amyloid-beta and Tau pathologies. Front Neurol 2018;9:549.

73. Lanctôt KL, Amatniek J, Ancoli-Israel S, Arnold SE, Ballard C, CohenMansfield J, et al. Neuropsychiatric signs and symptoms of Alzheimer's disease: New treatment paradigms. Alzheimers Dement (N Y) 2017; 3:440-449.

74. Xiao T, Zhang W, Jiao B, Pan C-Z, Liu X, Shen L. The role of exosomes in the pathogenesis of Alzheimer' disease. Transl Neurodegener 2017; 6:3.

75. Nhan HS, Chiang K, Koo EH. The multifaceted nature of amyloid precursor protein and its proteolytic fragments: friends and foes. Acta Neuropathol 2015;129:1-19.

76. Frost GR, Li YM. The role of astrocytes in amyloid production and Alzheimer's disease. Open Biol 2017;7.

77. Mudher A, Colin M, Dujardin S, Medina M, Dewachter I, Alavi Naini $\mathrm{SM}$, et al. What is the evidence that tau pathology spreads through prion-like propagation? Acta Neuropathol Commun 2017;5:99.

78. Yuyama K, Igarashi Y. Exosomes as carriers of Alzheimer's Amyloidss. Front Neurosci 2017;11:229.

79. Chen JJ, Zhao B, Zhao J, Li S. Potential roles of exosomal microRNAs as diagnostic biomarkers and therapeutic application in Alzheimer's disease. Neural Plasticity 2017;2017:7027380. 